\title{
Classical to quantum correspondence in dissipative directed transport
}

\author{
Gabriel G. Carlo, Alejandro M. F. Rivas, and María E. Spina \\ Departamento de Física, CNEA, Libertador 8250, (C1429BNP) Buenos Aires, Argentina
}

(Dated: October 30, 2015)

\begin{abstract}
We compare the quantum and classical properties of the (Quantum) Isoperiodic Stable Structures - (Q)ISSs - which organize the parameter space of a paradigmatic dissipative ratchet model, i.e. the dissipative modified kicked rotator. We study the spectral behavior of the corresponding classical Perron-Frobenius operators with thermal noise and the quantum superoperators without it for small $\hbar_{\text {eff }}$ values. We find a remarkable similarity between the classical and quantum spectra. This finding significantly extends previous results - obtained for the mean currents and asymptotic distributions only - and on the other hand unveils a classical to quantum correspondence mechanism where the classical noise is qualitatively different from the quantum one. This is crucial not only for simple attractors but also for chaotic ones, where just analyzing the asymptotic distribution reveals insufficient. Moreover, we provide with a detailed characterization of relevant eigenvectors by means of the corresponding Weyl-Wigner distributions, in order to better identify similarities and differences. Finally, this model being generic, it allows us to conjecture that this classical to quantum correspondence mechanism is a universal feature of dissipative systems.
\end{abstract}

PACS numbers: 05.45.Mt, 03.65.Yz, 05.60.Gg

\section{INTRODUCTION}

Research in directed transport has been steadily evolving since the main idea re-emerged in the 60's [1] to consolidate into a well established field nowadays [2-4]. Out of equilibrium spatiotemporal periodic systems are at the heart of these investigations, where the second law of thermodynamics no longer applies. Many disciplines have found the concept of ratchet transport very useful, they include such different areas as biology [5] on one end and nanotechnology [6] on the other. In chemistry, for example isomerization reactions have been recently characterized by directed transport properties [7]. Cold atoms $[8,9]$ and also Bose-Einstein condensates have been transported, these latter by means of quantum ratchet accelerators [10], where the current has no classical counterpart [11] and the energy grows ballistically $[12,13]$. This short list is incomplete and many other theoretical and experimental areas have been omitted here.

Breaking all spatiotemporal symmetries leading to momentum inversion [14] allows a net current. In particular, we are interested in deterministic ratchets with dissipation which have been historically associated with a classical asymmetric chaotic attractor [15]. Dissipative quantum ratchets, interesting for cold atoms experiments have been introduced in [16]. In recent works, the parameter space of the classical counterpart of this system has been studied in detail [17]. These new results have pointed out that not only chaotic attractors, but also several families of isoperiodic stable structures (ISSs, which are Lyapunov stable islands of different periods grouped into structures in parameter space, sometimes called "shrimps" due to their shape) have a fundamental role in understanding the current behavior. Moreover, they have been identified as a means to obtain optimal ratchet transport which is temperature resistant [18]. It is worth noticing that these ISSs are a common feature, found in generic dissipative dynamical systems. In fact, some of their properties have recently been studied in the dissipative kicked rotator model [19].

Their quantum manifestations, the so-called QISSs have been investigated in [20]. There it has been found that the QISSs look like the quantum chaotic attractors at their vicinity in parameter space (these corresponding to values of the parameters where the classical counterparts are already chaotic), with the exception of comparatively few cases. Recently, a complete parameter space picture has been obtained for the quantum system [21]. It was also shown in [20] that a thermal coarse-graining of the classical dynamical equations (i.e., adding thermal noise of the order of $\hbar_{\text {eff }}$ ) is sufficient to obtain a good approximation for the QISSs. In this paper we carry out a detailed spectral study of both the Perron-Frobenius operators associated with the classical evolution with thermal noise and the quantum superoperators without it, finding a great similarity between them. A known result in the decoherence literature [22] states that diffusive noise makes the quantum mechanical spectrum converge to that of the coarse grained Perron-Frobenius, the diffusive process being applied to both the classical and the quantum cases. Here, we find that for dissipative systems an effective way to reach the classical to quantum correspondence consists in applying a thermal (diffusive-like) noise solely at the classical level. We underline that this is not meant to be an example of the emergence of classicality [23]. It is important to say that in this work all the possible representative asymptotic cases have been considered, i.e. those in which the classical limiting set is either simple (in its main forms) or chaotic. Nevertheless a systematic study is left for the future. Finally, we study the similarities and differences of relevant eigenvectors with the help of Weyl-Wigner distributions in phase space. Thanks to this we are able to identify the limits of this correspondence mechanism. 
This paper is organized as follows: In Sec. II we present the system under study, i.e. a modified kicked rotator with dissipation and the methods we use to investigate it from the classical to quantum correspondence perspective. We introduce the Perron-Frobenius operator and the quantum superoperator. In Sec. III we analyze their spectra, showing the classical to quantum correspondence details. In Sec. IV we look at the behavior at the phase space level by means of the Weyl-Wigner distributions of the eigenvectors. Finally, in Sec. V we present our conclusions.

\section{MODEL AND CALCULATION METHODS}

We investigate the paradigmatic dissipative ratchet system given by the map $[16,18]$

$$
\left\{\begin{array}{l}
\bar{n}=\gamma n+k[\sin (x)+a \sin (2 x+\phi)] \\
\bar{x}=x+\tau \bar{n}
\end{array}\right.
$$

where $n$ is the momentum variable conjugated to $x, \tau$ is the period of the map and $\gamma$ is the dissipation parameter. This represents a particle moving in one dimension $[x \in(-\infty,+\infty)]$ under the influence of a periodic kicked asymmetric potential:

$$
V(x, t)=k\left[\cos (x)+\frac{a}{2} \cos (2 x+\phi)\right] \sum_{m=-\infty}^{+\infty} \delta(t-m \tau),
$$

where $\tau$ is the kicking period, also having a dissipation given by $0 \leq \gamma \leq 1$. When $\gamma=0$ the particle is in the overdamped regime and when $\gamma=1$ the evolution is conservative. Breaking the spatial $(a \neq 0$ and $\phi \neq m \pi)$ and temporal $(\gamma \neq 1)$ symmetries allows the net current generation. As is customary in this model the classical dynamics can be made dependent on the parameter $K=k \tau$ by means of introducing the rescaled momentum $p=\tau n$. In order to consider a thermal noise of the order of $\hbar_{\mathrm{eff}}$, the effective Planck constant which will be introduced in the quantum version, we simply change $\bar{n}^{\prime} \rightarrow \bar{n}$ in Eq. 1, where $\bar{n}^{\prime}=\bar{n}+\xi$. We can associate the noise variable $\xi$ with a temperature $T$ by means of the relation $<\xi^{2}>=2(1-\gamma) k_{B} T$, where $k_{B}$ is the Boltzmann constant (which we take equal to 1 ). The factor $(1-\gamma)$ has been taken to avoid having noise in the conservative limit, however many other choices are possible. Finally we have that $T \simeq \hbar_{\mathrm{eff}} /[2(1-\gamma)]$.

Quantizing this model (without thermal noise) is straightforward: $x \rightarrow \hat{x}, n \rightarrow \hat{n}=-i(d / d x)(\hbar=1)$. Since $[\hat{x}, \hat{p}]=i \tau($ where $\hat{p}=\tau \hat{n})$, the effective Planck constant is $\hbar_{\mathrm{eff}}=\tau$. The classical limit corresponds to $\hbar_{\mathrm{eff}} \rightarrow 0$, while $K=\hbar_{\text {eff }} k$ remains constant. Dissipation at the quantum level is introduced by means of the master equation [24] for the density operator $\hat{\rho}$ of the system

$$
\dot{\hat{\rho}}=-i\left[\hat{H}_{s}, \hat{\rho}\right]-\frac{1}{2} \sum_{\mu=1}^{2}\left\{\hat{L}_{\mu}^{\dagger} \hat{L}_{\mu}, \hat{\rho}\right\}+\sum_{\mu=1}^{2} \hat{L}_{\mu} \hat{\rho} \hat{L}_{\mu}^{\dagger} \equiv \Lambda \rho .
$$

Here $\hat{H}_{s}=\hat{n}^{2} / 2+V(\hat{x}, t)$ is the system Hamiltonian, $\{$, is the anticommutator, and $\hat{L}_{\mu}$ are the Lindblad operators given by $[25,26]$

$$
\begin{aligned}
& \hat{L}_{1}=g \sum_{n} \sqrt{n+1}|n\rangle\langle n+1|, \\
& \hat{L}_{2}=g \sum_{n} \sqrt{n+1}|-n\rangle\langle-n-1|,
\end{aligned}
$$

with $n=0,1, \ldots$ and $g=\sqrt{-\ln \gamma}$ (due to the Ehrenfest theorem).

The classical densities in phase space evolve with the Perron-Frobenius operator arising from the Liouville equation corresponding to the map (1). A coarse grained approximation to the Perron-Frobenius operator is obtained by means of the Ulam method [27], based on a discretization of the phase space. This method has been recently used to study dynamical maps very successfully [28]. To construct the Ulam matrix $S$, the phase space is divided into $M^{2}$ cells and then $n_{\text {tr }}$ random points from each cell $j$ are propagated according to the classical map. The elements $S_{i j}$ of the $M^{2} \times M^{2}$ matrix $S$ are given by $S_{i j}=\frac{n_{i j}}{n_{\mathrm{tr}}}$, where $n_{i j}$ is the number of trajectories arriving to cell $i$ from the cell $j$. This discretization introduces an effective diffusive noise of order $h_{\mathrm{eff}}^{\mathrm{PF}} \propto \frac{1}{M}$. For homogeneous systems and sufficiently large values of $M$ the Ulam method is expected to converge to the spectrum of the continuous system. When thermal noise is included in the classical calculation we have checked that the results obtained from the diagonalization are independent of the value of $h_{\mathrm{eff}}^{\mathrm{PF}}$ as long as $h_{\mathrm{eff}}^{\mathrm{PF}} \leq h_{\mathrm{eff}}$, i.e., the coarse graining inherent to the Ulam procedure is smaller than the thermal fluctuations. In the following we will refer to the Perron-Frobenius operator but the calculations are understood to be done with its Ulam approximation.

In the quantum case the evolution of the density matrix is given by $\rho_{t+1}=e^{\Lambda} \rho_{t}$, where $e^{\Lambda}$ is a non-unital superoperator of dimension $N^{2} \times N^{2}$ constructed by numerical integration of Eq. 3. Here $h_{\text {eff }} \propto \frac{1}{N}$.

For the diagonalization of $S$ (with and without thermal noise) and $e^{\Lambda}$ we have used the Arnoldi method, which allowed us to go to large dimensions, corresponding to $0.15<h_{\mathrm{eff}}^{\mathrm{PF}}<0.247$ for the classical matrix and $0.082<h_{\mathrm{eff}}<0.247$ for the quantum one. We should point out that these values mean diagonalizing matrices of a maximum size of $160000 \times 160000$ for the classical case (which takes longer to construct) and of $531441 \times 531441$ for the quantum case. Of course, we have compared similar $\hbar_{\mathrm{eff}}^{(\mathrm{PF})}$ sizes in both cases, expecting to improve the construction time for the classical matrix in the future, in order to compare with the already obtained quantum results.

\section{CLASSICAL TO QUANTUM CORRESPONDENCE FROM SPECTRA}

We will first look at the properties of the spectra of the Perron-Frobenius operator for the representative cases studied in [20]. These cases correspond to the three 


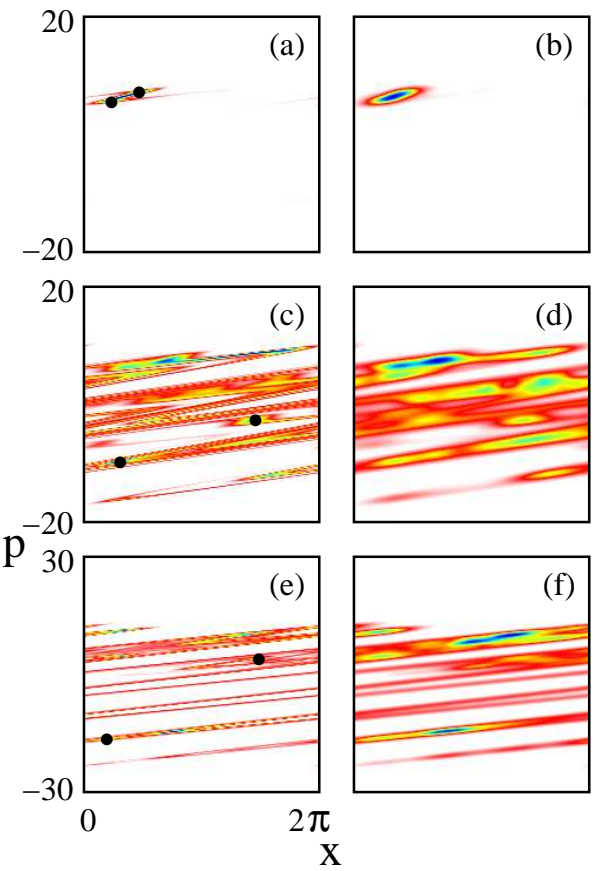

FIG. 1. (Color online) Phase space distributions corresponding to the ISSs labeled by $B_{1}(\gamma=0.2, k=8.2$, first row $), C_{-1}$ $(\gamma=0.64, k=5.6$, middle row $)$, and $D_{-1}(\gamma=0.29, k=11.9$, bottom row). Lower to higher values of these distributions go from white to (rainbow colors) grays. Left column shows the classical Poincaré maps while the right column shows the Husimi functions. In (a), (c), and (e) the periodic points of each ISS are marked by means of a black dot. The surrounding distributions correspond to the same ISSs with a thermal noise of the order of $\hbar_{\text {eff }}$. In (b), (d), and (f) $\hbar_{\text {eff }} \simeq 0.082$. Notice that for (e) and (f) $p \in[-30,30]$, while for the rest $p \in[-20,20]$. This Figure has been taken from [20].

main kinds of ISSs that were identified and called $B_{M}$, $C_{M}$ and $D_{M}$, where $M$ stands for an integer or rational number and corresponds to the mean momentum of these structures, in units of $2 \pi$. These structures are the main organizing features embedded in a chaotic background (named $A$ ) of the bidimensional parameter space given by the kick strength $k$, and the dissipation $\gamma$. We consider $B_{1}(k=8.2, \gamma=0.2$, the only one with an asymptotic positive current), $C_{-1}(k=5.6, \gamma=0.64)$, $D_{-1}(k=11.9, \gamma=0.29)$, and a chaotic attractor $(k=11.9, \gamma=0.26)$. The corresponding classical and quantum phase space distributions (with the exception of the chaotic attractor case, which is very similar to $\left.D_{-1}\right)$ can be seen in Fig. 1, which has been taken from [20]. It is worth mentioning that we take $a=0.5$ and $\phi=\pi / 2$ throughout this work.

In Fig. 2 we show the spectra of the Perron-Frobenius operator $S$ with no addition of thermal noise for two different values of the coarse graining: $\hbar_{\mathrm{eff}}^{\mathrm{PF}}=0.247$ and $\hbar_{\mathrm{eff}}^{\mathrm{PF}}=0.15$. By construction the spectra are contained
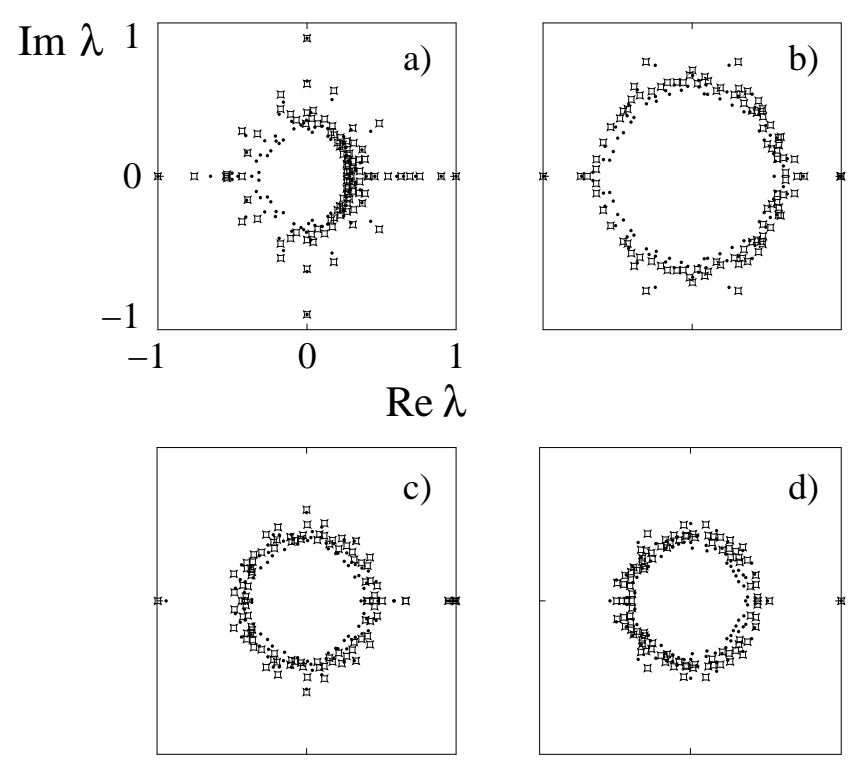

FIG. 2. (Color online) 100 largest eigenvalues of the PerronFrobenius operator $S$ for the a) $B_{1}$, b) $C_{-1}$, c) $D_{-1}$, and d) chaotic attractor cases. (Blue) gray squares correspond to $\hbar_{\mathrm{eff}}^{\mathrm{PF}}=0.15$, while black dots to $\hbar_{\mathrm{eff}}^{\mathrm{PF}}=0.247$.

in the unit circle and have a non-degenerate eigenvalue $\lambda_{0}=1$ [29]. The right eigenvectors corresponding to $\lambda_{0}$ should reproduce the structure of the asymptotic classical distributions previously found (see Fig. 1), which are point like ISS's in cases a), b) and c), and a fractal chaotic attractor in $\mathrm{d}$ ). We have checked that this is indeed the case, except for a small discrepancy at the lowest resolution for case $D_{-1}$, where the point like structure is slightly embedded in a distribution resembling the neighboring chaotic attractor case. This suggests that in this region of the parameter space $\hbar_{\mathrm{eff}}^{\mathrm{PF}}=0.247$ is not sufficient to resolve neighboring maps with different properties, and one has to go to larger matrix dimensions. As can be seen from the Figure, the leading eigenvalues remain approximately fixed as $\hbar_{\text {eff }}^{\mathrm{PF}}$ decreases. This stability reflects the convergence of the Ulam procedure.

For the ISSs cases, $\lambda_{1}$ (the eigenvalue following $\lambda_{0}$ in decreasing order of their moduli) is very close to 1 in accordance with the very long times required for the equilibration of these structures. As can be seen from Table I the decay times $t_{\lambda_{1}}$ given essentially by the value of the spectral gap $1-\left|\lambda_{1}\right|$, are in fairly good agreement with the equilibration times obtained in [20] by means of the ratchet currents. Again, we notice that in the $D_{-1}$ case the time $t_{\lambda_{1}}$ characterizing the decay towards the complex invariant state, although large, is substantially shorter than the equilibration time of about 700 steps obtained with the exact classical dynamics [20].

In Fig. 3 the quantum spectra for $\hbar_{\mathrm{eff}}=0.15$ ((red) gray squares) are shown together with the classical results obtained from the diagonalization of the Perron Frobenius operator with a thermal noise satisfying < $\xi^{2}>=\hbar_{\mathrm{eff}}$ (black dots). Comparing with Fig. 2 it is 


\begin{tabular}{|c|cc|cc|cc|}
\cline { 2 - 7 } \multicolumn{1}{c|}{} & \multicolumn{2}{c|}{$P F$} & \multicolumn{2}{c|}{$P F_{\text {th }}$} & \multicolumn{2}{c|}{$Q M$} \\
\cline { 2 - 7 } \multicolumn{1}{c|}{} & $\lambda_{1}$ & $t_{\lambda_{1}}$ & $\lambda_{1}$ & $t_{\lambda_{1}}$ & $\lambda_{1}$ & $t_{\lambda_{1}}$ \\
\hline$B_{-1}$ & 0.900 & 43.7 & 0.840 & 26.4 & 0.818 & 22.9 \\
\hline$C_{-1}$ & 0.994 & 765.2 & 0.727 & 14.4 & 0.701 & 13.0 \\
\hline$D_{-1}$ & 0.992 & 573.3 & 0.449 & 5.7 & 0.376 & 4.7 \\
\hline $\operatorname{attr}$ & 0.523 & 7.1 & 0.452 & 5.8 & 0.410 & 5.2 \\
\hline
\end{tabular}

TABLE I. Perron-Frobenius $\lambda_{1}$ values and equilibration times $t_{\lambda_{1}}$ (defined by $\lambda_{1}^{t_{\lambda_{1}}}=0.01$ ) are shown in columns 1 and 2 for all 4 cases analyzed in the main text. Columns 3 and 4 show the same for the Perron-Frobenius with thermal noise, and columns 5 and 6 for the quantum mechanical case. We take $\hbar_{\mathrm{eff}}^{(\mathrm{PF})}=\hbar_{\mathrm{eff}}=0.15$.
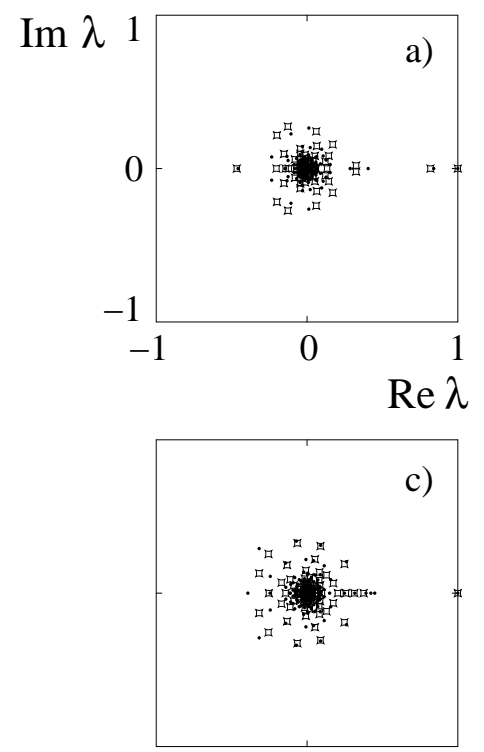

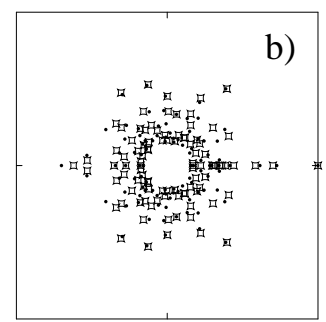

b)

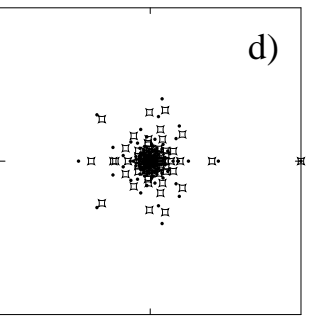

FIG. 3. (Color online) 100 largest eigenvalues for the quantum superoperator $e^{\Lambda}$ and for the Perron-Frobenius operator with thermal noise $S^{\text {thermal }}$ for the a) $B_{1}$, b) $C_{-1}$, c) $D_{-1}$, and d) chaotic attractor cases. (Red) gray squares correspond to the quantum model, while black dots to the classical one. $\hbar_{\mathrm{eff}}^{(\mathrm{PF})}=\hbar_{\mathrm{eff}}=0.15$.

clear that the bare coarse graining (even the greatest one $\left.\hbar_{\mathrm{eff}}^{\mathrm{PF}}=0.247\right)$ involved in the Ulam procedure is not enough to reproduce the quantum features in any of the studied ISSs, not even in the $D_{-1}$ nor in the attractor case. The inclusion of the temperature makes the classical spectra change dramatically in all cases, becoming remarkably similar to those of the quantum system for the set of eigenvalues of modulus closer to 1. Even so, it should be noticed that the quantum dynamics is more contractive in spectral terms than the classical one. In fact, a huge fraction of the quantum eigenvalues have absolute values very close to 0 . This was already pointed out in [30] for the dissipative baker map, where the differences between a full quantization and a discretization procedure were studied.

In all cases the spectral gap "jumps" to the "correct" quantum mechanical one. This is a highly non-trivial effect that underlines the classical and quantum similari- ties. The chaotic attractor case presents a singular property, this being that the value of the quantum mechanical $\lambda_{1}$ is in good agreement with its classical counterparts, with or without thermal noise. Therefore the $t_{\lambda_{1}}$ is about the same in all cases (see last row of Table I) confirming the results of Fig. 4 of [20] which shows that in the case of the chaotic attractor, classical and quantum currents have similar equilibration times. For the remaining eigenvalues the correspondence is not exact, but the similarities are evident.

We observe that the spectra of $D_{-1}$ and the chaotic attractor cases are very close. This deepens on the hypothesis that chaotic attractors could be approximated by neighboring ISSs, suggesting that the approximation could be extended to the superoperator itself. As a matter of fact, from these results we can conjecture that the correspondence is at the (super) operator level and that there should exist an approximate formal equivalence between them, at least in their long-lived sector, i.e. the block associated to the largest eigenvalues. In the following we will identify some limits to this conjecture.

\section{THE PHASE SPACE PICTURE}

In order to give a complementary point of view to analyze this classical to quantum correspondence mechanism we study the phase space behavior. We will compare the right eigenvectors of the Perron-Frobenius operator with thermal noise, with the Weyl-Wigner symbol for the right eigenvectors of the quantum superoperator. Regarding the former it is worth noting that the invariant eigenstates (with $\lambda=1$ ) are real and non negative, in agreement with the Perron-Frobenius theorem, and can be interpreted as probability distributions in phase space. The remaining eigenvectors are real (although non positive) for eigenvalues on the real axis and complex otherwise. In the quantum case the invariant eigenstates have the property of being density matrices with $\operatorname{Tr}\left(\hat{R}_{\lambda_{0}}\right)=1$, while the remaining ones are traceless.

The Weyl-Wigner representation improves on previous results obtained by using Husimi distributions [20], since we are able to appreciate interference fringes (coherences) and finer details of the quantum eigenvectors. Weyl-Wigner symbols for a $N$ dimensional Hilbert space are defined in a redundant $2 N \times 2 N$ discrete phase space [31]. This is formed by the grid of points $x=\frac{1}{N}(a, b)$ with $a$ and $b$ semi integer numbers running from 0 to $N-1 / 2$. In this way, the Weyl-Wigner symbol $R(x)$ of the operator $\hat{R}$ is obtained from its matrix elements in the coordinate representation as

$$
R(x)=\sum_{n=0}^{N-1}<q_{2 b-n}|\hat{R}| q_{n}>\exp \left(\frac{i 2 \pi}{N} 2 a(b-n)\right) .
$$

In order to get rid of redundancies and "ghost images" derived essentially from the cylindrical topology of our phase space, we use a method that has been developed 
by Argüelles and Dittrich [32] consisting of Fourier transforming the Weyl-Wigner symbol to its symplectic analogue, known as the "chord symbol". Then, after performing a cut off for the longer chords and antifourier transforming, the new Weyl-Wigner symbol with the desired properties is obtained. This latter is supported by a $N \times N$ discrete phase space formed by points $x=\frac{1}{N}(i, j)$ where now $i$ and $j$ are integer numbers running from 0 to $N-1$. This is the symbol we use in the following.

It is important noticing that the eigenvectors are defined within a global phase factor. For the invariant eigenstates the property of being a density matrix ensures that the Weyl-Wigner symbol is real. However, differently from the classical case, the quantum symbols display negative values due to interference fringes. Indeed as it has been shown in [33] for dissipative Markovian systems in the semiclassical limit, any initial pure Wigner function evolves into a positive-definite phase space distribution. For the remaining traceless eigenvectors, adjusting the phase factor, we obtained that, in accordance with the classical case, the Weyl-Wigner symbols are real for eigenvalues on the real axis and complex otherwise. The complex eigenvalues come in complex conjugated pairs. Their corresponding eigenvectors form pairs of complex conjugated distributions in phase space also, both for the classical and the quantum case.

In Table II we compare the overlaps between the WeylWigner symbol of the eigenvectors of the quantum superoperator with the corresponding eigenvectors of the Perron-Frobenius operator with thermal noise. We do this up to the 5th pair in decreasing order of the moduli of their corresponding eigenvalues. To calculate these overlaps we take into account that any state $\hat{R}$ can be represented by $R(x)$ with $x=(p, q)$ a point in phase space. For the classical states, $R(x)$ stands for the right eigenvector, while for the quantum ones, $R(x)$ is the WeylWigner symbol. Hence, given any two states $\hat{R}_{1}$ and $\hat{R}_{2}$ , their overlap is defined as:

$$
\begin{gathered}
O\left(\hat{R}_{1}, \hat{R}_{2}\right)=\operatorname{Tr}\left(\hat{R}_{1} \hat{R}_{2}\right) / \sqrt{\left[\operatorname{Tr}\left(\hat{R}_{1}^{2}\right) \operatorname{Tr}\left(\hat{R}_{2}^{2}\right)\right]}= \\
\sum_{x} R_{1}(x) R_{2}^{*}(x) / \sqrt{\left[\left(\sum_{x}\left|R_{1}(x)\right|^{2}\right)\left(\sum_{x}\left|R_{2}(x)\right|^{2}\right)\right]}
\end{gathered}
$$

where $R^{*}(x)$ and $|R(x)|$ stand respectively for the complex conjugate and absolute value of $R(x)$. The overlap defined above is a complex magnitude, its modulus is invariant even though its argument depends on the relative phase between the eigenvectors. Also, when this relative phase is null $O\left(\hat{R}_{1}, \hat{R}_{2}\right)$ is real. In all cases the value of the overlap is about 0.9 for the invariant states and progressively decreases as we go to smaller $|\lambda|$.

In the following Figures we display the phase space portraits of selected eigenvectors of the Perron-Frobenius operator with thermal noise and the Weyl-Wigner symbols of the corresponding eigenvector of the quantum superoperator.

\begin{tabular}{|c|c|c|c|c|}
\hline & $B_{-1}$ & $C_{1}$ & $D_{1}$ & Attr \\
\hline \hline$\lambda_{0}$ & 0.9449 & 0.9349 & 0.8697 & 0.8654 \\
$\lambda_{1}$ & 0.9441 & 0.8132 & 0.5422 & 0.5186 \\
$\lambda_{2}$ & 0.5622 & 0.8504 & 0.6381 & 0.6689 \\
$\lambda_{3}$ & 0.5681 & 0.8505 & 0.6382 & 0.6689 \\
$\lambda_{4}$ & 0.4421 & 0.8141 & 0.5190 & 0.4178 \\
\hline
\end{tabular}

TABLE II. Overlaps of the first 5 eigenvectors of the quantum superoperator with the corresponding eigenvectors of the Perron-Frobenius operator with thermal noise, for all cases.
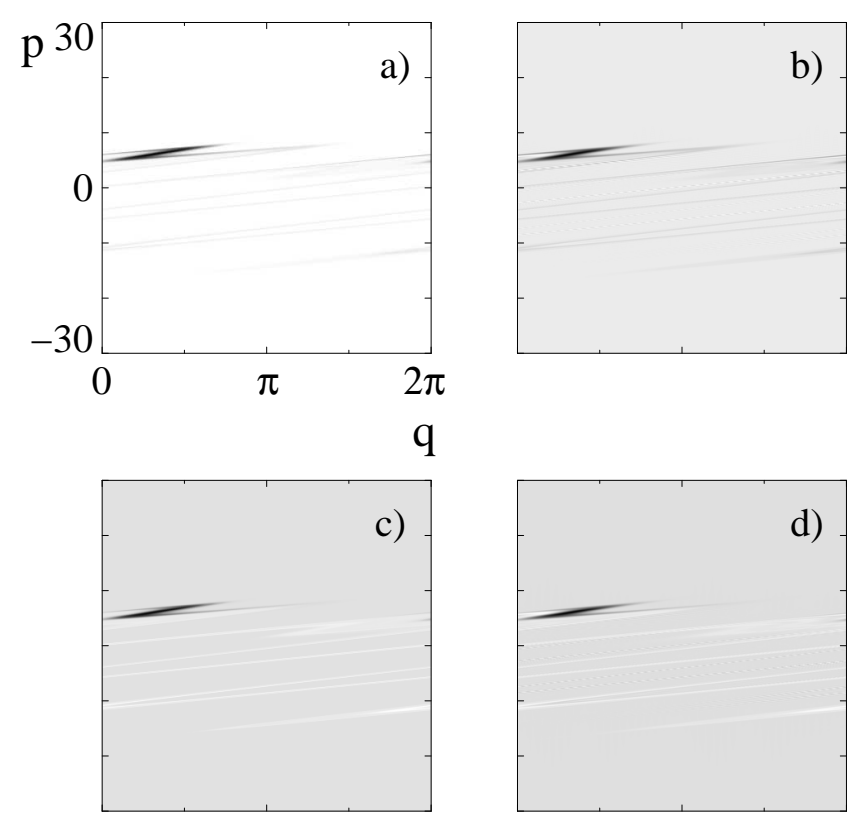

FIG. 4. Phase space portraits of two eigenvectors of the Perron-Frobenius with thermal noise (left panels) and the Weyl-Wigner symbols for the corresponding quantum eigenstates (right panels), for the $B_{1}$ ISS. In a) and b) we show the eigenvectors associated with $\lambda_{0}$, while in c) and d) the ones corresponding to $\lambda_{1}\left(\lambda_{1}=0.840\right.$ in the classical spectrum and $\lambda_{1}=0.818$ in the quantum one). In all cases $\hbar_{\mathrm{eff}}^{(\mathrm{PF})}=0.15$.

We first analyze the $B_{1}$ ISS. This is the only case where the invariant distributions shown in Fig.4 a) and b) correspond to point-like attractors, even though some traces of the chaotic basin of attractor are visible in both panels. As can be seen from Fig.4 a) a smearing out of the order of $h_{\text {eff }}$ is enough to turn the classical points corresponding to the ISS into a distribution of exactly the same shape of the quantum one depicted in Fig. 4 b). The quantum-classical similarity is also striking for the 2nd eigenvector pair displayed in Fig.4 c) and d) . Despite both having a negative image of the basin of attraction these states behave in a very similar way to the invariant pair.

For the next case corresponding to the $C_{-1}$ ISS, we have chosen to show the details of the eigenvectors associated with complex $\lambda_{2}$. The real parts shown in Fig. 5 a) and b) are different from the imaginary ones shown in Fig. $5 \mathrm{c}$ ) and d), and again the coincidence between 

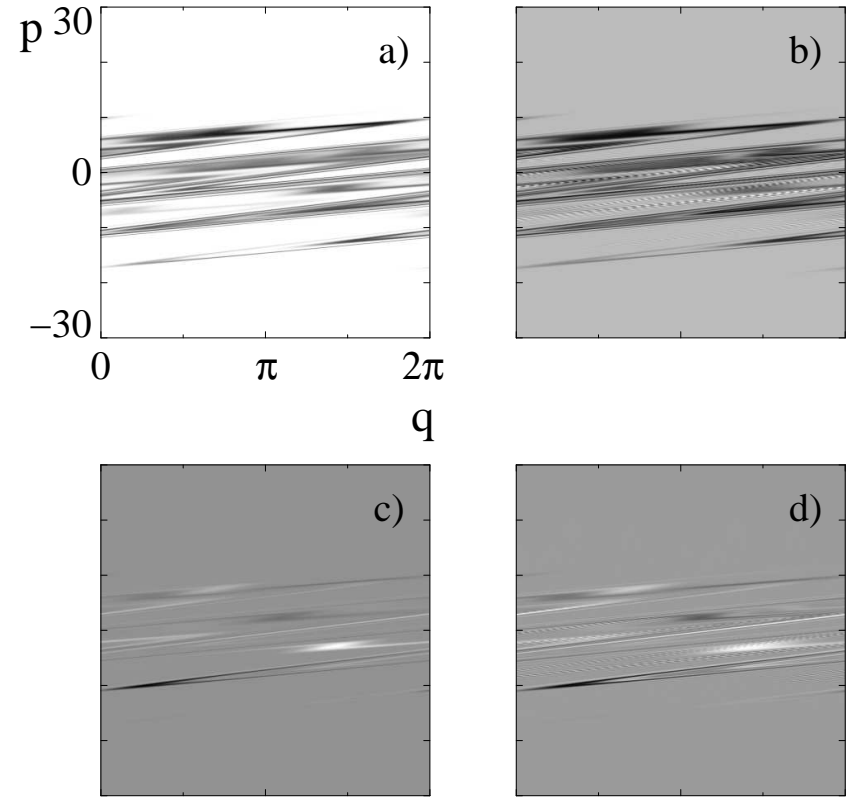

FIG. 5. Phase space portrait of the eigenvector associated with the complex eigenvalue $\lambda_{2}\left(\lambda_{2}=0.388+\mathrm{i} 0.500\right.$ in the classical spectrum and $\lambda_{2}=0.396+\mathrm{i} 0.501$ in the quantum one) of the Perron Frobenius with thermal noise (left panels) and the Weyl-Wigner symbols for the corresponding quantum eigenstates (right panels) for the $C_{-1}$ ISS. In a) and b) we show the real part of the distributions while in c) and d) the imaginary one. In all cases $\hbar_{\text {eff }}^{(\mathrm{PF})}=0.15$.

classical and quantum phase space portraits is remarkable. This is also clearly reflected in the value of the overlap. The main difference is in the presence of interference fringes in the quantum case which do not have enough weight to spoil the overlap in a significant way.

The next two cases are better analyzed together since they belong to the same region of the parameter space. It can be seen that they coincide, having very similar invariant states shown in Figs. 6 and 7, panels a) and b). The differences are only present through interference fringes at the quantum level. But they are clearly not very relevant, both looking at these representations and also at the values of the overlaps. On the other hand, we show the eigenvectors corresponding to the worst performing overlaps in both cases in Figs. 6 and 7, panels c) and d). They show that in these cases the coherences have a greater weight. Anyway if we look carefully at the distributions they are quite alike for the remaining of the distributions. This puts a limit on the previously mentioned hypothesis consisting of approximating the long lived block of the quantum superoperators with the Perron-Frobenius ones with thermal noise. A quantitative study is left for the future.
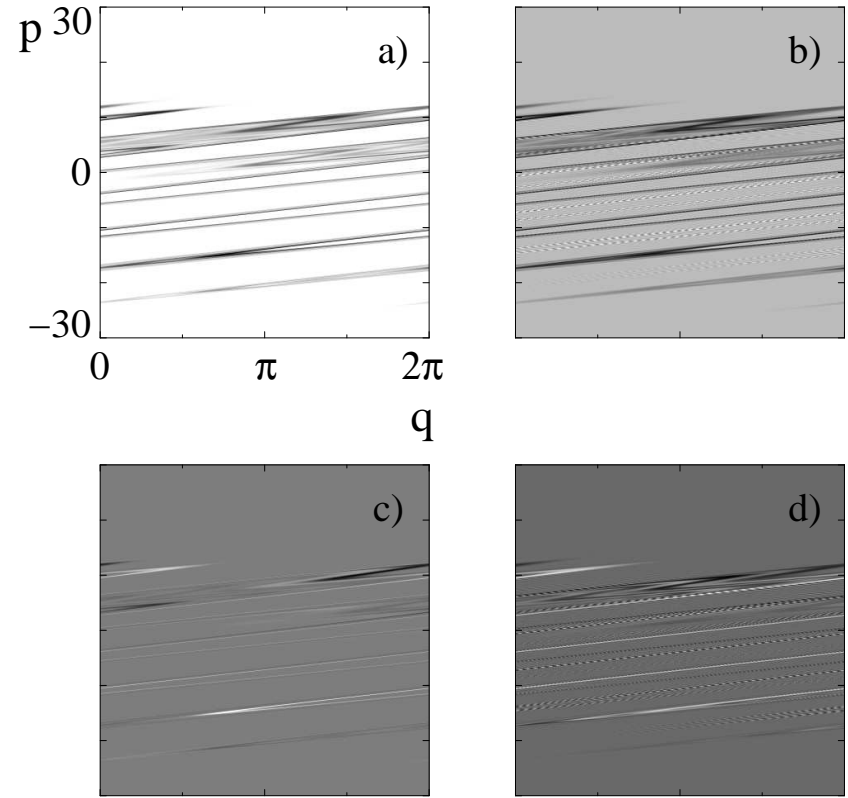

FIG. 6. Phase space portraits of two eigenvectors of the Perron-Frobenius with thermal noise (left panels) and the Weyl-Wigner symbols for the corresponding quantum eigenstates (right panels), for the $D_{-1}$ ISS. In a) and b) we show the eigenvectors associated with $\lambda_{0}$, while in c) and d) the ones corresponding to $\lambda_{4}\left(\lambda_{4}=0.391\right.$ in the classical spectrum and $\lambda_{4}=0.313$ in the quantum one). In all cases $\hbar_{\mathrm{eff}}^{(\mathrm{PF})}=0.15$.
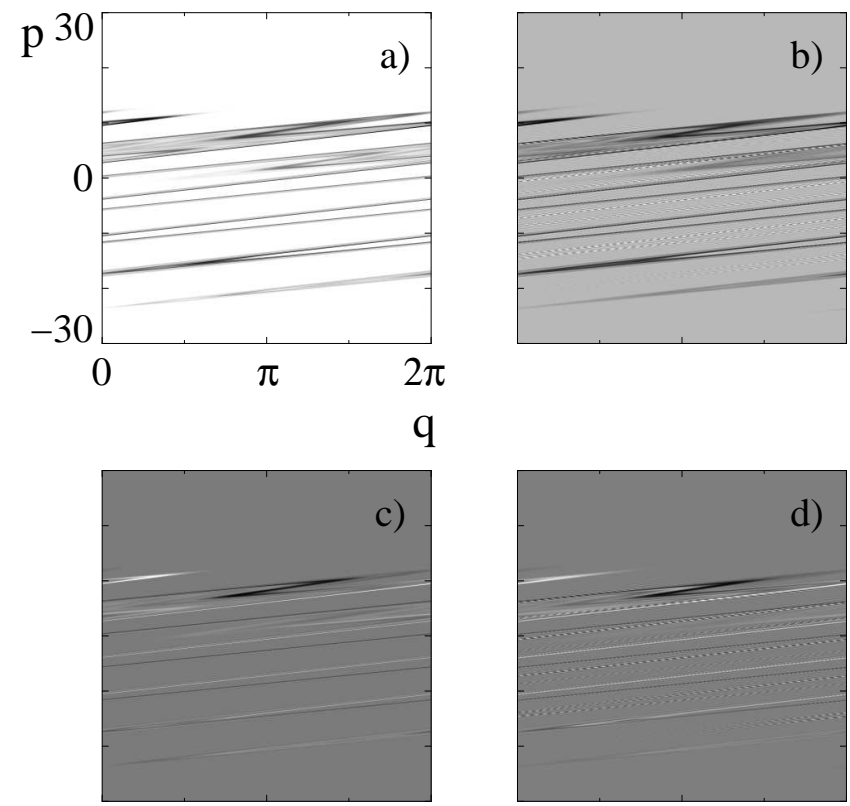

FIG. 7. Phase space portraits of two eigenvectors of the Perron-Frobenius with thermal noise (left panels) and the Weyl-Wigner symbols for the corresponding quantum eigenstates (right panels), for the chaotic attractor. In a) and b) we show the eigenvectors associated with $\lambda_{0}$, while in c) and d) the ones corresponding to $\lambda_{4}\left(\lambda_{4}=-0.471\right.$ in the classical spectrum and $\lambda_{4}=-0.391$ in the quantum one). In all cases $\hbar_{\mathrm{eff}}^{(\mathrm{PF})}=0.15$. 


\section{CONCLUSIONS}

By studying the behavior of the spectra and eigenvectors of both the Perron-Frobenius operators with thermal noise and the quantum mechanical superoperators associated with the ISSs and chaotic attractors typical of a paradigmatic model for dissipative directed transport, we were able to identify a novel mechanism of classical to quantum correspondence hinted in [20] (just looking at the ratchet currents and asymptotic distributions). The remarkable coincidence of both sets of eigenvalues is indicative of an approximate formal equivalence between the classical and quantum realms. In the following we raise some important points derived from this result.

Firstly, it is interesting to link our approach with previous works. In [34] the dissipative kicked top model was analyzed, with a special focus in the case where it decays to a chaotic attractor. There it was found that the quantum invariant state closely follows the structure of the classical attractor, without the finer details. It was also shown that in the semiclassical limit the leading eigenvalues of the quantum spectrum converge to the ones of the Perron-Frobenius, thus implying that the quantum mechanical time scales become independent of $h_{\text {eff }}$ and coincide with time scales set by the Ruelle resonances. In the present work the classical to quantum correspondence is investigated in representative regions of the parameter space of a paradigmatic dissipative ratchet system, corresponding to chaotic attractors and to ISSs as well. Besides, we put the accent on small but finite $\hbar_{\text {eff }}$ values, for which we find a correspondence mechanism based on adding thermal noise at the classical level only. Our approach is also clearly distinct from the usual way in which the quantum to classical correspondence is treated in the decoherence literature where the procedure consists in applying a diffusive noise to both the classical and the quantum equations in order for the spectra to converge to each other [22]. To summarize, our classical approximation to a dissipative quantum system is not in conflict with decoherence theory, neither with semiclassical approximations. It is just a very simple and effective way to obtain a classical to quantum correspondence for finite $\hbar_{\mathrm{eff}}$.

On the other hand, the consequences of this finding are relevant not only to directed transport but to dissipative systems in general. A very promising one is the possibility to approximate the quantum asymptotic state in the chaotic case by the classical dynamics with thermal noise corresponding to a simple attractor in its vicinity. This was previously conjectured in [20] but now with the help of the Weyl-Wigner distributions we are able to show that this is indeed possible. As a matter of fact, the differences due to coherences are minor. Moreover, it is worth noticing that even when having a classical chaotic attractor the addition of thermal noise at this level is necessary in order to find a spectrum similar to the quantum one. Hence, studying these effects could be interesting for many applications as for example in superconducting quantum interference devices (SQUIDs) [35].

Finally, we would like to point out that the long-lived sector of the quantum superoperators corresponding to any kind of structure in parameter space could be well approximated by means of Perron-Frobenius operators with thermal noise. This conclusion can be drawn from the remarkable similarity among the spectra for all the cases shown in Sec. III. However, in our way to devise a classical approximation we have found a problem hardly visible using just Husimi distributions as in [20]. Thanks to the Weyl-Wigner representations of Sec. IV we have found that the eigenvectors, even some long-lived ones, can have differences due to the coherences remaining in the quantum system. Despite this, their weight seems to be not so important. This keeps our hopes to find an effective approximation theory in the future.

\section{ACKNOWLEDGMENTS}

Support from CONICET is gratefully acknowledged.
[1] R. P. Feynman, Lectures on Physics, Vol. 1, (AddisonWesley, Reading, MA, 1963).

[2] P. Reimann, Phys. Rep. 361, 57 (2002).

[3] S. Kohler, J. Lehmann, and P. Hänggi, Phys. Rep. 406, 379 (2005).

[4] S. Denisov, S. Flach, and P. Hänggi, Phys. Rep. 538, 77 (2014).

[5] F. Jülicher, A. Ajdari and J. Prost, Rev. Mod. Phys. 69, 1269 (1997); G. Mahmud et al., Nature Phys. 5, 606 (2009); G. Lambert, D. Liao, and R.H. Austin, Phys. Rev. Lett. 104, 168102 (2010).

[6] R. D. Astumian, Science 276, 917 (1997); D. Reguera, A. Luque, P.S. Burada, G. Schmid, J.M. Rubí,and P.
Hänggi, Phys. Rev. Lett. 108, 020604 (2012).

[7] J. B. Gong and P. Brumer, Annu. Rev. Phys. Chem. 56, 1 (2005); G. G. Carlo, L. Ermann, F. Borondo, and R. M. Benito, Phys. Rev. E 83, 011103 (2011).

[8] P. H. Jones, M. Goonasekera, D.R. Meacher, T. Jonckheere, and T.S. Monteiro, Phys. Rev. Lett. 98, 073002 (2007); T. Salger, S. Kling, T. Hecking, C. Geckeler, L. Morales-Molina, and M. Weitz, Science 326, 1241 (2009).

[9] T. S. Monteiro, P. A. Dando, N. A. C. Hutchings, and M. R. Isherwood, Phys. Rev. Lett. 89, 194102 (2002); G. G. Carlo, G. Benenti, G. Casati, S. Wimberger, O. Morsch, R. Mannella, and E. Arimondo, Phys. Rev. A 74, 033617 (2006). 
[10] M. Sadgrove, M. Horikoshi, T. Sekimura, and K. Nakagawa, Phys. Rev. Lett. 99, 043002 (2007); I. Dana, V. Ramareddy, I. Talukdar, and G.S. Summy, Phys. Rev. Lett. 100, 024103 (2008).

[11] E. Lundh and M. Wallin, Phys. Rev. Lett. 94, 110603 (2005); D. Poletti, G. G. Carlo, and B. Li, Phys. Rev. E 75, 011102 (2007).

[12] A. Kenfack, J. Gong, and A.K. Pattanayak, Phys. Rev. Lett. 100, 044104 (2008); J. Wang and J. Gong, Phys. Rev. E 78, 036219 (2008).

[13] M. Sadgrove, M. Horikoshi, T. Sekimura, and K. Nakagawa, Eur. Phys. J. D 45, 229 (2007).

[14] S. Flach, O. Yevtushenko, and Y. Zolotaryuk, Phys. Rev. Lett. 84, 2358 (2000).

[15] J. L. Mateos, Phys. Rev. Lett 84, 258 (2000).

[16] G. G. Carlo, G. Benenti, G. Casati, and D.L. Shepelyansky, Phys. Rev. Lett. 94, 164101 (2005).

[17] A. Celestino, C. Manchein, H.A. Albuquerque, and M.W. Beims, Phys. Rev. Lett. 106, 234101 (2011).

[18] C. Manchein, A. Celestino, and M.W. Beims, Phys. Rev. Lett. 110, 114102 (2013).

[19] D.F.M. Oliveira, M. Robnik, and E.D. Leone, Chaos 21, 043122 (2011).

[20] G.G. Carlo, Phys. Rev. Lett. 108, 210605 (2012).

[21] L. Ermann and G.G. Carlo, Phys. Rev. E 91, 010903(R) (2015).
[22] S. Nonnenmacher, Nonlinearity 16, 1685 (2003).

[23] W.H. Zurek, Rev. Mod. Phys. 75, 715 (2003).

[24] G. Lindblad, Commun. Math. Phys. 48, 119 (1976).

[25] T. Dittrich and R. Graham, Europhys. Lett., 7, 287 (1988).

[26] R. Graham, Z. Phys. B Cond. Mat., 59, 75 (1985).

[27] A collection of mathematical problems, S.M. Ulam, Interscience tracts in pure and applied mathematics 8, Interscience, New York, 1960.

[28] L. Ermann and D.L. Shepelyansky, Eur. Phys. J. B, 75, 299 (2010); K.M. Frahm, and D.L. Shepelyansky, Eur. Phys. J. B, 76, 57 (2010); L. Ermann, K.M. Frahm, and D.L. Shepelyansky, arXiv:1409.0428 (2014).

[29] Introduction to Dynamical Systems, M. Brin and G. Stuck, Cambridge University Press, Cambridge UK, 2002.

[30] G. G. Carlo, A. M. F. Rivas, and M. E. Spina, Phys. Rev. E 84, 066201 (2011).

[31] A.M.F. Rivas and A.M. Ozorio de Almeida, Annals of Physics 276, 223, (1999).

[32] A. Argüelles and T. Dittrich, Physica A 356, 72, (2005).

[33] A.M. Ozorio de Almeida, P. de M. Rios and O. Brodier, J. Phys. A: Math. Theor. 42, 065306 (2009).

[34] Dissipative Quantum Chaos and Decoherence, D. Braun, Springer Tracts in Modern Physics 172, Springer, Berlin Heidelberg New York, 2001.

[35] M.J. Everitt, New J. of Phys. 11, 013014 (2009). 